\begin{tabular}{|c|c|}
\hline Title & $\begin{array}{l}\text { Possible Involvement of Phosphatidylinositol 3-Kinase, but Not Protein Kinase B or Glycogen Synthase Kinase } 3 \beta \text {, in } \\
\text { Progesterone Induced Oocyte Maturation in the Japanese Brown Frog, Ranajaponica }\end{array}$ \\
\hline Author(s) & Ota, Ryoma; Suwa, Kaori; Kotani, Tomoya; Mita, Koich; Y amashita, Masakane \\
\hline Citation & $\begin{array}{l}\text { Zoological Science, 25(7), } 773.781 \\
\text { https://doi.org/10.2108/2sj.25.773 }\end{array}$ \\
\hline Issue Date & $2008-07$ \\
\hline DOC URL & http:/hdl.handle.net/2115/39623 \\
\hline Rights & (c) Zoological Society of Japan /本文献の公開は著者の意思に基づくものである \\
\hline Type & article \\
\hline File Information & zsj25p773.pdf \\
\hline
\end{tabular}

Instructions for use 


\title{
Possible Involvement of Phosphatidylinositol 3-Kinase, but Not Protein Kinase B or Glycogen Synthase Kinase 3 $\beta$, in Progesterone-Induced Oocyte Maturation in the Japanese Brown Frog, Rana japonica
}

\author{
Ryoma Ota, Kaori Suwa ${ }^{\dagger}$, Tomoya Kotani, Koich Mita ${ }^{\ddagger}$ \\ and Masakane Yamashita* \\ Laboratory of Reproductive and Developmental Biology, Faculty of Advanced \\ Life Science, Hokkaido University, Sapporo 060-0810, Japan
}

\begin{abstract}
It is known that amphibian oocytes undergo maturation through the formation and activation of maturation-promoting factor (MPF) in response to stimulation by the maturation-inducing hormone progesterone; however, the signal transduction pathway that links the hormonal stimulation on the oocyte surface to the activation of MPF in the oocyte cytoplasm remains a mystery. The aim of this study was to investigate whether the signal transduction mediated by phosphatidylinositol 3-kinase (PI3K), protein kinase B (PKB), and glycogen synthase kinase $3 \beta$ (GSK3 $\beta$ ) is involved in progesterone-induced oocyte maturation in the Japanese brown frog, Rana japonica. Inhibitors of PI3K, wortmannin and LY294002, inhibited progesterone-stimulated germinal vesicle breakdown (GVBD) only when the oocytes were treated at the initial phase of maturation, suggesting that PI3K is involved in the progesterone-induced maturation of Rana oocytes. However, we also obtained results suggesting that PKB and GSK3 $\beta$ are not involved in Rana oocyte maturation. A constitutively active PKB expressed in the oocytes failed to induce GVBD in the absence of progesterone despite its high level of kinase activity. A Myc-tagged PKB expressed in the oocytes (used to monitor endogenous PKB activity) was not activated in the process of progesterone-induced oocyte maturation. Overexpression of GSK3 $\beta$, which is reported to retard the progress of Xenopus oocyte maturation, had no effect on Rana oocyte maturation. On the basis of these results, we propose that PI3K is involved in the initiation of Rana oocyte maturation, but that neither PKB nor GSK3 $\beta$ is a component of the PI3K signal transduction pathway.
\end{abstract}

Key words: amphibians, oocyte maturation, phosphatidylinositol 3-kinase, progesterone, signal transduction

\section{INTRODUCTION}

Oocytes are produced in ovaries by the entry of mitotically proliferating oogonia into meiosis. Oocytes stop their meiotic cell cycle at the first prophase, during which they grow by the accumulation of substances necessary for early embryonic development. In many species, fully grown postvitellogenic oocytes arrested at the first meiotic prophase are immature, and they are unable to be fertilized until they mature (Masui, 1985). Oocyte maturation is induced by sequential actions of three substances: gonadotropic hormone (GTH), maturation-inducing hormone $(\mathrm{MIH})$, and maturation-promoting factor (MPF) (Nagahama

\footnotetext{
* Corresponding author. Phone: +81-11-706-4454;

Fax : +81-11-706-4456;

E-mail: myama@sci.hokudai.ac.jp

† Present address: Medico-Legal Section, Criminal Investigation Laboratory, Metropolitan Police Department, Tokyo 100-8929, Japan

‡ Present address: Faculty of Liberal Arts, Tokushima Bunri University, Sanuki, Tokushima 769-2193, Japan

doi: $10.2108 / z$ sj.25.773
}

et al., 1995; Yamashita, 2000; Nagahama and Yamashita, 2008). Oocytes that have been induced to mature resume meiosis from the first prophase and proceed to the first or second metaphase, at which time, in many invertebrates and vertebrates, they are inseminated. During the course of maturation, oocytes undergo drastic morphological changes associated with progression of the meiotic cell cycle, among which breakdown of the oocyte nuclear envelope (germinal vesicle breakdown, GVBD) occurring at the prophase/ metaphase transition is frequently regarded as a hallmark of the progress of maturation (Yamashita, 1998).

Amphibian oocytes provide the most widely used experimental system for investigating the molecular and cellular mechanisms of oocyte maturation, as these oocytes are ready induced to mature in vitro when treated with the $\mathrm{MIH}$ progesterone and are available in large numbers and sizes appropriate for experimental manipulation (Kotani et al., 2001; Kotani and Yamashita, 2002). It is known that the MIH signal received on the oocyte surface is transduced into the oocyte cytoplasm to form and activate MPF; however, actual biochemical pathways that link the hormonal stimulation to 
the activation of MPF are still not clear (Nakahata et al., 2003; Haccard and Jessus, 2006).

MPF consists of the catalytic subunit Cdc2 and the regulatory subunit Cyclin B. Although its function in promoting metaphase is common among species, the formation and activation mechanisms of MPF differ among species (Yamashita et al., 2000). In Xenopus, immature oocytes contain inactive MPF, called pre-MPF, which consists of Cyclin B-bound Cdc2 phosphorylated on both T14/Y15 and T161. Progesterone stimulates dephosphorylation of T14/ Y15, yielding active MPF that consists of Cyclin B-bound Cdc2 phosphorylated only on T161. In contrast to Xenopus, pre-MPF is absent in immature Rana oocytes. After stimulation by progesterone, Cyclin B is synthesized from its stored mRNA and binds to the preexisting Cdc2. Then CAK phosphorylates Cyclin B-bound Cdc2 on T161. Neither T14/Y15 phosphorylation nor its dephosphorylation is involved in MPF activation in Rana (Ihara et al., 1998). Other amphibians except Xenopus probably utilize similar mechanisms (Tanaka and Yamashita, 1995; Sakamoto et al., 1998).

In accordance with MPF activation, many other kinases are activated during amphibian oocyte maturation. These include the Mos-MEK-MAPK pathway (MAPK is a mitogenactivated protein kinase and MEK is a MAPK/ERK kinase.) and p70 ribosomal S6 kinase (p70 s6k: Ferrell, 1999; Nebreda and Ferby, 2000). We previously demonstrated that the Mos/MAPK pathway is not essential for MPF activation and GVBD during oocyte maturation in Rana japonica (Yoshida et al., 2000a), while in Xenopus, activation and inhibition of the Mos/MAPK pathway result in the initiation and the inhibition of oocyte maturation, respectively (Palmer and Nebreda, 2000). Moreover, it has been reported that inhibition of $\mathrm{p} 70^{\mathrm{s} 6 \mathrm{k}}$ by rapamycin prevents GVBD in Rana dybowskii but not in Xenopus (Bandyopadhyay et al., 1999). Thus, there is a difference between Rana and Xenopus in the signal transduction pathways used in oocyte maturation stimulated by progesterone, in addition to the difference in the molecular mechanisms of MPF activation.

Ju et al. (2002) have suggested that phosphatidylinositol 3-kinase (PI3K) is involved in progesterone-induced oocyte maturation in Rana dybowskii. A downstream mediator of $\mathrm{PI} 3 \mathrm{~K}$ is protein kinase $\mathrm{B}(\mathrm{PKB})$, and $\mathrm{PKB}$ in turn phosphorylates and inactivates glycogen synthase kinase $3 \beta$ (GSK3 $\beta$; Cross et al., 1995), which is required for Xenopus oocyte maturation (Fisher et al., 1999). It is therefore likely that a signal transduction pathway mediated by PI3K/PKB/GSK3 $\beta$ plays a role in oocyte maturation common in amphibians. However, there is little information on PKB and GSK3 $\beta$ in Rana oocyte maturation. In this study, we demonstrated inhibition of progesterone-induced maturation of Rana japonica with inhibitors specific to PI3K, suggesting the invovement of PI3K in initiation of Rana oocyte maturation. However, a constitutively active PKB failed to induce GVBD in Rana oocytes despite its high level of kinase activity. The kinase activity of PKB did not remarkably change during progesterone-stimulated oocyte maturation. Moreover, overexpression of GSK3 $\beta$ had no apparent effect on Rana oocyte maturation, although it retarded the time course of Xenopus oocyte maturation as previously reported (Fisher et al., 1999). On the basis of these findings, we propose that $\mathrm{PI} 3 \mathrm{~K}$, but not PKB or GSK3 $\beta$, is essential for initiating Rana oocyte maturation in response to progesterone.

\section{MATERIALS AND METHODS}

Induction of oocyte maturation and its inhibition with PI3K inhibitors

Sexually mature Japanese brown frogs, Rana japonica, were purchased from a dealer just before hibernation and stored at $4{ }^{\circ} \mathrm{C}$ until the natural breeding season. Immature oocytes were isolated with collagenase as described previously (Ihara et al., 1998). All of the experiments were carried out at room temperature unless otherwise noted. The oocytes were treated with $10 \mu \mathrm{M}$ progesterone for 30 min in modified Barth's saline buffered with HEPES (MBS-H; Cyert and Kirschner, 1988). The presence of GVBD was examined by cutting the oocytes with a razor blade.

PI3K inhibitors, wortmannin (SIGMA, Tokyo, Japan) and LY294002 (SIGMA), were dissolved in dimethyl sulfoxide (DMSO, SIGMA) as stock solution ( $10 \mathrm{mM}$ ) and then diluted with MBS-H to yield appropriate concentrations before use. Following treatment of oocytes with wortmannin or LY294002, progesterone was applied to the oocytes to induce maturation. To determine the period for which wortmannin is effective, oocytes pretreated with $1 \mu \mathrm{M}$ wortmannin and $10 \mu \mathrm{M}$ progesterone for 30 min were washed three times with MBS-H to remove the drugs. The oocytes were further incubated in the continuous presence of $1 \mu \mathrm{M}$ wortmannin, starting at $3 \mathrm{~h}$ after the washing.

\section{Isolation of cDNA encoding Xenopus laevis PKB and GSK3 $\beta$}

Total RNA was isolated from the Xenopus ovary with ISOGEN (Nippon Gene, Tokyo, Japan). Following precipitation with $\mathrm{LiCl}$, Poly $(A)+$ RNA was isolated by the use of oligo(dT)-Latex beads (Oligotex-dT30<Super>, Roche, Tokyo, Japan), and cDNAs were produced by using a First-Strand cDNA Synthesis Kit (Gibco BRL LIFE Technology, Tokyo, Japan). According to the published sequences, the ORFs of Xenopus PKB and GSK3 $\beta$ (DDBJ/EMBL/ GenBank database accession numbers AF317656 and L38492, respectively) were amplified by RT-PCR using the following oligonucleotide primers in which were introduced $\mathrm{BamHI}$ and $\mathrm{Xhol}$ sites for PKB and BamHI and HindIII sites for GSK3 $\beta$ as indicated by underlines: for Xenopus PKB, XI PKB-F primer, 5'-AAGGATCCATGAATGAAGTAGCGATAGTG-3', and XI PKB-R primer, 5'-TTTCTCGAG-

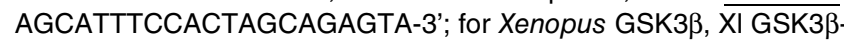
F primer, 5'-CGGGATCCATGAGCGGAAGGCCGAGAACC-3', and XI GSK3 $\beta$-R primer, 5'-CCCAAGCTTGGAGGAGTTGGAGGCAGATGC3'. The PCR product of Xenopus PKB was digested with BamHI and $X h o l$ and was then ligated into the same cloning sites of pBluescript II SK(+) (Stratagene, La Jolla, CA). After sequence confirmation, the insert was isolated by cutting the plasmid with BamHI and Xhol and was then ligated into pET21a (Novagen, Madison, WI). The PCR product of Xenopus GSK3 $\beta$ was digested with BamHI and HindIII and was then ligated into the same cloning sites of pET21a.

\section{Production of anti-PKB antibody}

Recombinant Xenopus PKB was expressed in E. coli BL21(DE3)pLysS. In contrast to the molecular size (56 kDa) expected from its cDNA sequence, the recombinant protein was expressed as a $45-\mathrm{kDa}$ protein in $E$. coli. Sequencing of the $\mathrm{N}$ terminal amino acids revealed that the protein lacked $178 \mathrm{~N}$ terminal amino acids, starting from methionine 179 (data not shown). The bacterially produced protein was purified by sodium dodecyl sulfate-polyacrylamide gel electrophoresis (SDS-PAGE), followed by electroelution in Tris-glycine buffer without SDS, according to the method described previously (Hirai et al., 1992). The purified protein was dialyzed against $1 \mathrm{mM}$ HEPES $(\mathrm{pH} 7.0)$, lyophilized, and injected into two mice to produce antibodies as described previously (Yamashita et al., 1991). Polyclonal antibodies against the recombinant PKB were affinity-purified with the anti- 
genic proteins electroblotted onto an Immobilon membrane (Millipore, Tokyo, Japan). The antibodies obtained from the two mice showed similar specificities.

\section{Immunoblotting}

Oocytes were washed three times with ice-cold extraction buffer (EB: $100 \mathrm{mM} \beta$-glycerophosphate, $20 \mathrm{mM}$ HEPES, $15 \mathrm{mM}$ $\mathrm{MgCl}_{2}, 5 \mathrm{mM}$ EGTA, $1 \mathrm{mM}$ dithiothreitol, $100 \mu \mathrm{M}$ ( $p$-amidinophenyl) methanesulfonyl fluoride, $3 \mu \mathrm{g} / \mu$ l leupeptin, $\mathrm{pH}$ 7.5). After removing excess EB with filter paper, one volume of new EB was added. The oocytes were homogenized with a pestle (Pellet Pestle, Kontes, Vineland, NJ) and centrifuged at $10,000 \times \mathrm{g}$ for $10 \mathrm{~min}$ at $4^{\circ} \mathrm{C}$. The supernatant was collected and stored at $-80^{\circ} \mathrm{C}$.

The oocyte extracts were subjected to SDS-PAGE, and separated proteins were transferred to an Immobilon membrane. The blots were probed with the following antibodies: anti-Xenopus PKB mouse polyclonal antibody cross-reactive with Rana PKB (this study), anti-Xenopus GSK3 $\beta$ mouse monoclonal antibody crossreactive with Rana GSK3 $\beta$ (Santa Cruz Biotechnology), and antiMyc mouse monoclonal antibody (9E10). The antigen-antibody complex was visualized by alkaline phosphatase-conjugated secondary antibodies, as previously described (Yamashita et al., 1991).

\section{Production of mutant PKB and GSK3 $\beta$}

Two mutant forms of Xenopus PKB were produced in this study: TS PKB, in which two active phosphorylation sites, threonine 309 (T309) and serine 474 (S474), were mutated to glutamic acid (T309E and S474E, respectively) to make a constitutively active kinase; and KTS PKB, in which lysine 180 (K180) in the kinase domain of TS PKB was mutated to alanine (K180A) to make a kinase-deficient version of TS PKB. To make a kinase-deficient version of GSK3 $\beta$, lysine 85 in the kinase domain of GSK3 $\beta$ was mutated to methionine (K85M). To construct these mutants, the pBluescript plasmid containing the ORF of Xenopus PKB and the pET21a plasmid containing the ORF of Xenopus GSK3 $\beta$ were mutated by using a QuickChange SiteDirected Mutagenesis Kit (Stratagene) and the following oligonucleotide sets (the newly introduced restriction enzyme site in each mutation is underlined.): for XI PKB K180R, 5'-GGACGATATTACGCTATGAGAATTCTAAAAAAAGAAGTTATTGTTGC-3' and 5'-GCAACAATAACTTCTTTTTTTAGAATTCTCATAGCGTAATATCGTCC-3'; for XI PKB T309E, 5'-GGAATAAAAGATGGAGCAACCATGAAGGAATTCTGTGGAACACC and 5'-GGTGTTCCACAGAATTCCTTCATGGTTGCTCCATCTTTTATTCC-3'; for XI PKB S474E, 5'-CCGCACTTCCCCCAGTTTGAATATTCTGCTAGTGGAAATGC-3' and 5'GCATTTCCACTAGCAGAATATTCAAACTGGGGGAAGTGCGG-3'; for XI GSK3 $\beta$ K85M, 5'-GAGCTGGTGGCTATCATGAAGGTGCTGCAAGAC-3' and 5'-GTCTTGCAGCACCTTCATGATAGCCACCAGCTC-3'. Mutated clones were first selected by the restriction enzyme site newly introduced and then confirmed by DNA sequencing. In addition to these mutants, we also produced $\triangle \mathrm{PH} T \mathrm{TS} \mathrm{PKB}$ and $\triangle \mathrm{PH}$ KTS PKB in which the pleckstrin homology $(\mathrm{PH})$ domain was removed from TS PKB and KTS PKB, respectively. To do this, 100 $\mathrm{N}$-terminal amino acids were eliminated from Xenopus $\mathrm{PKB}$ proteins (wild-type PKB, TS PKB, and KTS PKB) by PCR using an XI PKB11-100 primer (5'-AAGGATCCAAGAAGCAGGAAGAAGAAATG-3') and $\mathrm{XI}$ PKB-R primer, followed by ligation of the PCR products into the BamHI/Xhol site of pBluescript II SK (+).

Expression of wild-type and mutant PKB proteins in oocytes was performed by injecting in vitro transcribed $\mathrm{mRNA}$ encoding each PKB tagged with the Myc-epitope (EQKLISEEDL), which can be recognized by the anti-Myc mouse monoclonal antibody 9E10. To construct Myc-tagged PKB proteins, we first produced an expression vector for introducing the Myc-tag into the $\mathrm{N}$ terminus of target protein (Myc/pCS2+) as follows: 1) Two oligonucleotides including the Myc-epitope sequence (Myc-F, 5'-GAAGATCTACCATGGCATCAATGCAGAAGCTGATCTCAGAGGAGGACCTGGGATCCA-3') and its complimentary sequence (Myc-R, 5'-TGGATCCCAGGTCCTCCTC-
TGAGATCAGCTTCTGCATTGATGCCATGGTAGATCTTC-3') were annealed, and the resulting DNA was treated with $B g / l I$ and $B a m H I$, 2) after gel-filtration with Sephadex G-50 (Amersham Biosciences, Tokyo, Japan) to remove the digested ends of DNA, the DNA was ligated into the $\mathrm{BamHI}$ site of pCS2+, and 3) the correctly oriented clone was selected by DNA sequencing. We then cut all of the plasmids containing wild-type PKB (WT PKB), TS PKB, KTS PKB, $\triangle \mathrm{PH} T S \mathrm{PKB}$, and $\triangle \mathrm{PH}$ KTS PKB with $\mathrm{BamHI} / \mathrm{Xhol}$, and ligated the resulting inserts into the $\mathrm{BamHI} / \mathrm{Xhol}$ site of $\mathrm{Myc} / \mathrm{pCS} 2+$ to produce Myc-tagged PKB proteins (Myc-WT PKB, Myc-TS PKB, Myc-KTS PKB, Myc- $\Delta$ PH TS PKB, and Myc- $\triangle \mathrm{PH}$ KTS PKB).

\section{Microinjection into oocytes}

Fully grown immature Rana oocytes were manually isolated from the ovary with fine forceps and stored in MBS-H. For mRNA injection, the plasmids carrying PKBs and GSK3 $\beta$ s were linearized with $A p a l$ and $B s p E I$, respectively, mRNAs encoding PKB were transcribed by using an mMESSAGE mMACHINE SP6 Kit (Ambion, Austin, TX), and mRNAs encoding GSK3 $\beta$ were transcribed by using an mMESSAGE mMACHINE T7 Ultra Kit (Ambion). All in vitro synthesized mRNAs were phenol/chloroform-extracted, isopropanolprecipitated, and dissolved in distilled water. Isolated oocytes were injected with $50 \mathrm{nl}$ of $1 \mathrm{ng} / \mathrm{nl}$ mRNAs encoding PKB and $0.1 \mathrm{ng} / \mathrm{nl}$ mRNAs encoding GSK3 $\beta$. After injection, the oocytes were incubated overnight at $18^{\circ} \mathrm{C}$, treated with progesterone, and examined for the presence of GVBD at appropriate times.

\section{PKB kinase assays}

For measuring PKB activity, oocytes were injected with $50 \mathrm{ng}$ of Myc-WT PKB mRNA and incubated overnight at $18^{\circ} \mathrm{C}$ to allow the oocytes to express the protein (five oocytes were extracted to confirm the expression of PKB by immunoblotting.). The oocytes were then treated with progesterone and, at intervals of $3 \mathrm{~h}, 10$ oocytes were extracted with lysis buffer $(20 \mathrm{mM}$ Tris- $\mathrm{HCl}, 137 \mathrm{mM}$ $\mathrm{NaCl}, 1 \mathrm{mM}$ sodium orthovanadate, $100 \mathrm{mM} \mathrm{NaF}, 10 \mathrm{mM}$ sodium pyrophosphate, $1 \mathrm{mM}$ EGTA, $15 \mathrm{mM} \mathrm{MgCl}$, $1 \%$ TritonX-100, 10\% glycerol, $\mathrm{pH}$ 7.4) (Hehl et al., 2001). As a blank for PKB kinase activity, extracts from 10 uninjected oocytes were also obtained. The extracts were subjected to immunoprecipitation with anti-Myc antibody, and PKB activity associated with the immunoprecipitates was assayed in vitro by using a synthetic peptide (AKTide-2T, ARKRERTYSFGHHA) (Obata et al., 2000). A reaction buffer $(45 \mu \mathrm{l})$ containing $20 \mathrm{mM}$ HEPES, $\mathrm{pH} 7.4,0.5 \mathrm{mM}$ ATP with $1.5 \mu \mathrm{Ci}\left[\gamma^{-32} \mathrm{P}\right]$ ATP, $10 \mathrm{mM}$ dithiothreitol, $10 \mathrm{mM} \mathrm{MgCl}$, $0.5 \mathrm{mM}$ EGTA, and 0.1 $\mathrm{mM}$ AKTide-2T was added to the immunoprecipitates, and after incubation at room temperature for $20 \mathrm{~min}$, the kinase reaction was terminated by adding $5 \mu \mathrm{l}$ of $3 \mathrm{M}$ phosphoric acid. Following a brief centrifugation to settle the immunoprecipitates, the supernatants were spotted onto Whatman P81 phosphocellulose paper. The paper was washed three times in $1 \%$ phosphoric acid, and the radioactivity remaining on the paper was measured by scintillation counting. In each measurement, the value obtained from mRNAuninjected oocytes was subtracted from those obtained from mRNA-injected oocytes as a blank. After measuring the kinase activity, the immunoprecipitates were subjected to immunoblotting to confirm the constant protein content of Myc-WT PKB in each sample.

\section{RESULTS}

\section{Effects of PI3K inhibitors on progesterone-induced oocyte maturation}

Based on the finding that progesterone-induced oocyte maturation in Rana dybowskii is inhibited by inhibitors specific for PI3K, wortmannin and LY294002, the involvement of $\mathrm{PI} 3 \mathrm{~K}$ in oocyte maturation in this species has been suggested (Ju et al., 2002). In this study, we first investigated 

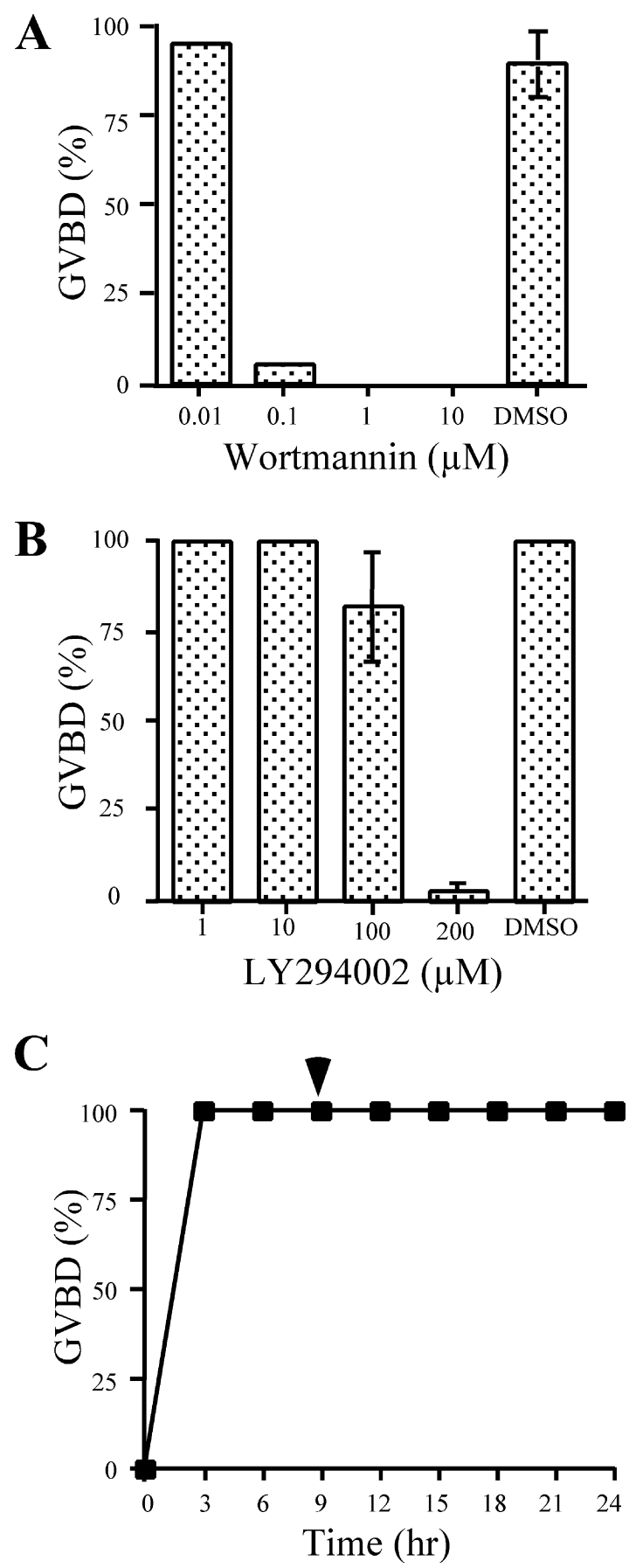

Fig. 1. Inhibition of progesterone-induced oocyte maturation by PI3K inhibitors. (A) Oocytes were incubated in the presence of 10 $\mu \mathrm{M}$ progesterone and the indicated concentration of wortmannin or $0.1 \%$ DMSO as a vehicle control. The oocytes were examined for the presence of GVBD after $24 \mathrm{~h}$. Each value is the mean \pm SEM of values from three experiments. (B) Oocytes were incubated with 10 $\mu \mathrm{M}$ progesterone and the indicated concentration of LY294002 or $2 \%$ DMSO as a vehicle control. (C) The period during which wortmannin had an effect on oocyte maturation. After the oocytes had been treated with $10 \mu \mathrm{M}$ progesterone and $1 \mu \mathrm{M}$ wortmannin for 30 min and had been washed with MBS-H to remove the drugs, $1 \mu \mathrm{M}$ wortmannin was added at 3-h intervals. The oocytes were examined for the presence of GVBD $25 \mathrm{~h}$ after progesterone treatment. The initiation of GVBD is indicated by an arrowhead. Note that wortmannin was effective only when added within $3 \mathrm{~h}$ after the washing.
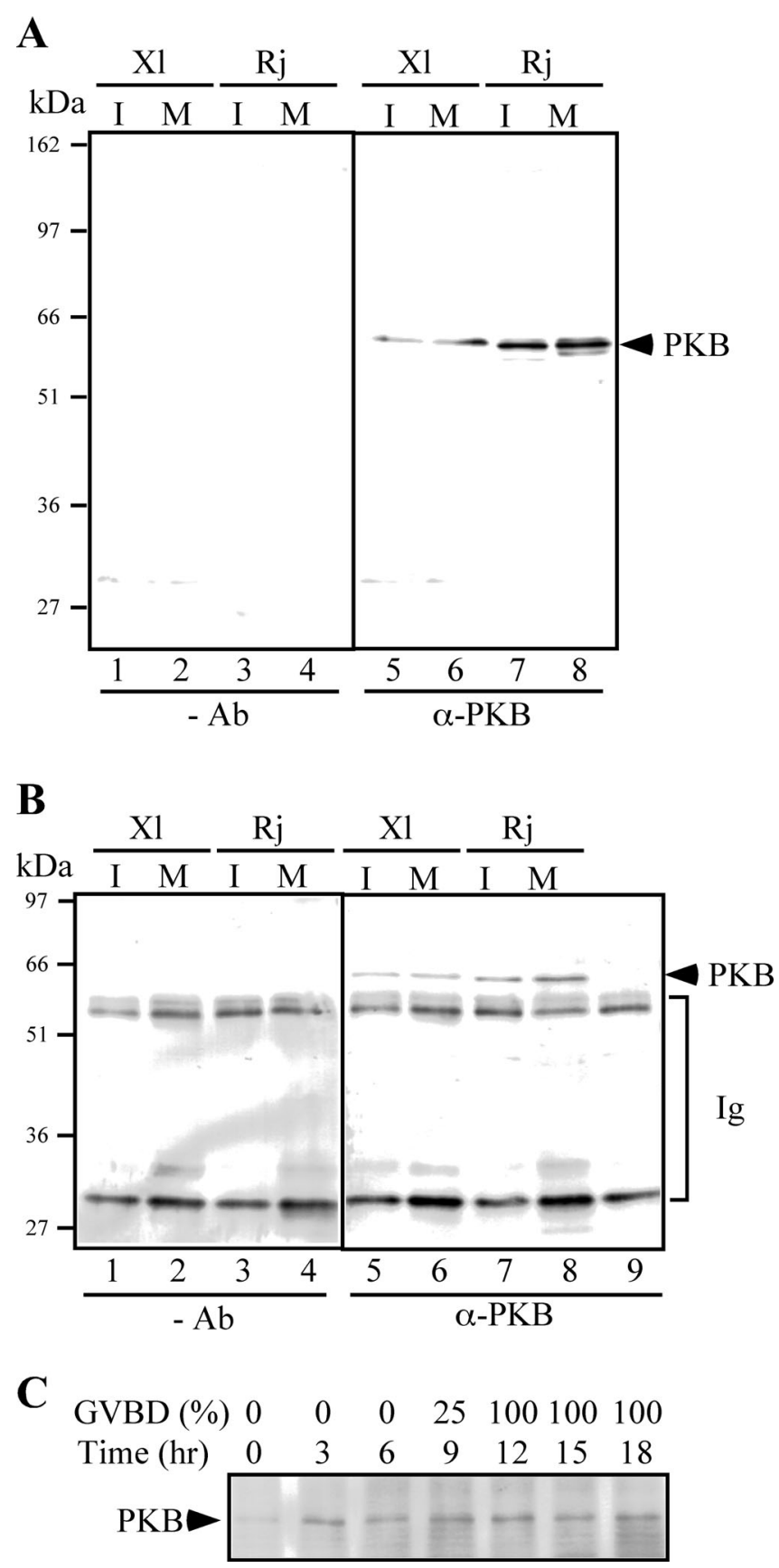

Fig. 2. Characterization of anti-PKB antibody by immunoblotting. (A) Anti-PKB immunoblots of crude extracts from immature (I) and mature (M) oocytes of Xenopus laevis (XI) (lanes 5 and 6) and Rana japonica (Rj) (lanes 7 and 8). Blots incubated without the primary antibody are also shown as controls (lanes 1-4). (B) Anti-PKB immunoblots of anti-PKB immunoprecipitates from immature (I) and mature (M) oocyte extracts of Xenopus laevis (XI) (lanes 5 and 6) and Rana japonica (Rj) (lanes 7 and 8). Blots incubated without the primary antibody are also shown as controls (lanes 1-4). Anti-PKB immunoprecipitate without oocyte extract (lane 9) shows the positions of immunoglobulins (Ig). (C) Anti-PKB immunoblots of crude extracts from Rana oocytes treated with $10 \mu \mathrm{M}$ progesterone, showing no remarkable change in PKB protein content during oocyte maturation. 
whether similar inhibition of oocyte maturation is observed in Rana japonica. Both wortmannin (Fig. 1A) and LY294002 (Fig. 1B) inhibited progesterone-induced GVBD in a dosedependent manner, although the effective dose of LY294002 (100-200 $\mu \mathrm{M})$ was higher than that of wortmannin $(0.1-10 \mu \mathrm{M})$. The period for which wortmannin was effective was limited to the early phase of progesteroneinduced oocyte maturation in Rana japonica (Fig. 1C), consistent with results obtained in Rana dybowskii (Ju et al., 2002).

\section{PKB during progesterone-induced oocyte maturation}

The inhibition by wortmannin and LY294002 suggests the involvement of PI3K in progesterone-induce oocyte maturation in Rana. Although it is still not clear what kinases function immediately downstream of PI3K during oocyte maturation, PKB is generally known as a downstream mediator of PI3K (Coffer et al., 1998). We therefore investigated the involvement of $\mathrm{PKB}$ in progesterone-induced oocyte

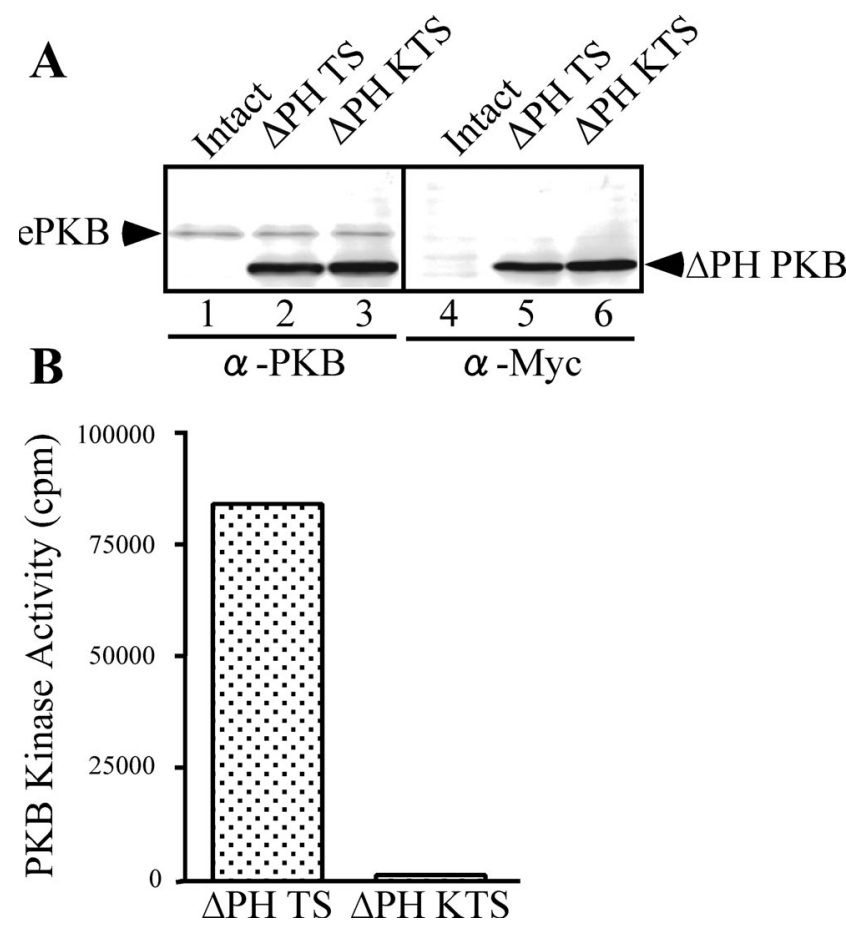

Fig. 3. Expression of constitutively active PKB in oocytes. (A) Confirmation of mutant PKB expression by immunoblotting. Oocytes were injected with mRNA encoding either Myc- $\triangle \mathrm{PH}$ TS PKB or Myc$\triangle \mathrm{PH} K T S \mathrm{PKB}$, incubated overnight, and extracted for immunoblotting. Crude extracts from uninjected immature oocytes (Intact), $\triangle \mathrm{PH}$ TS-injected oocytes ( $\triangle \mathrm{PH} T S)$, and $\Delta \mathrm{PH} K T S$-injected oocytes $(\Delta \mathrm{PH}$ KTS) were probed with anti-PKB antibody (lanes 1-3) and anti-Myc antibody (lanes 4-6). Endogenous PKB (ePKB), as well as the expressed $\triangle \mathrm{PH}$ PKB, was detected by anti-PKB antibody (lanes 13). (B) Kinase activity of mutant PKB proteins expressed in the oocytes. Oocytes injected with mRNA encoding either Myc- $\Delta \mathrm{PH}$ TS PKB or Myc- $\triangle \mathrm{PH}$ KTS PKB were incubated overnight. Extracts from 10 oocytes were immunoprecipitated with anti-Myc antibody, and the associated PKB kinase activity was measured. The value obtained from uninjected oocyte extract was subtracted from each value as a blank. Representative data are shown. Similar results were obtained repeatedly. maturation in Rana japonica. We first investigated whether PKB inhibitors, Akt (PKB) inhibitor \#1 and NL-71-101 (Calbiochem), have inhibitory effects on Rana oocyte maturation, but these drugs did not inhibit Rana oocyte maturation (data not shown).

To gain insight into the function of PKB in Rana oocyte maturation, we intended to examine the behavior of $\mathrm{PKB}$ during oocyte maturation by immunological analyses. However, a preliminary experiment showed that commercially available antibodies against mammalian PKB (Phospho-Akt [Ser 473] antibodies, \#4051 and \#9271; Cell Signaling Technology, Beverly, MA) are not cross-reactive with Rana japonica PKB (data not shown). We therefore decided to try to raise a new antibody against amphibian PKB. According to a cDNA sequence of Xenopus laevis PKB, we produced recombinant $\mathrm{PKB}$ in $E$. coli and used it for producing an antibody against amphibian PKB in mice.

The newly produced antibody was characterized by immunoblotting extracts from immature and mature oocytes of Xenopus laevis and Rana japonica. In both species (Fig. $2 \mathrm{~A}$ ), the anti-PKB antibody specifically recognized a $60-\mathrm{kDa}$

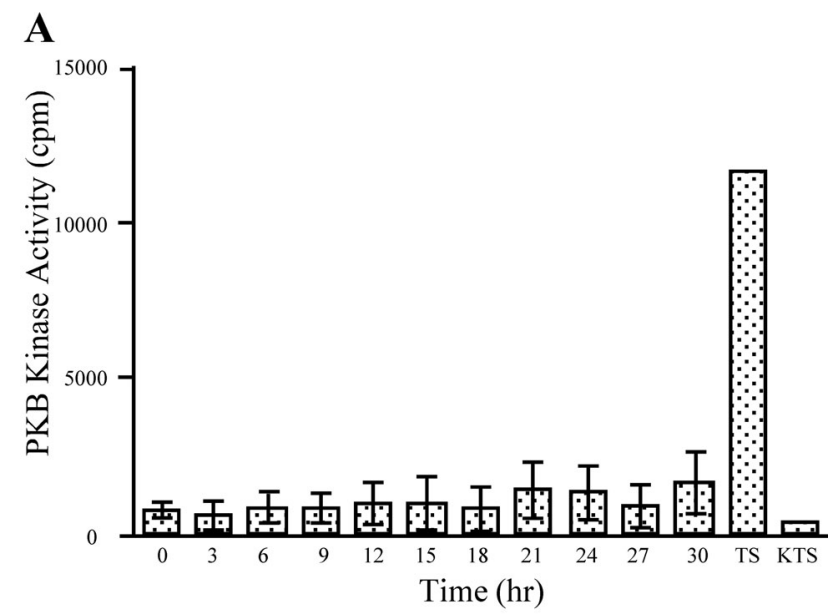

B

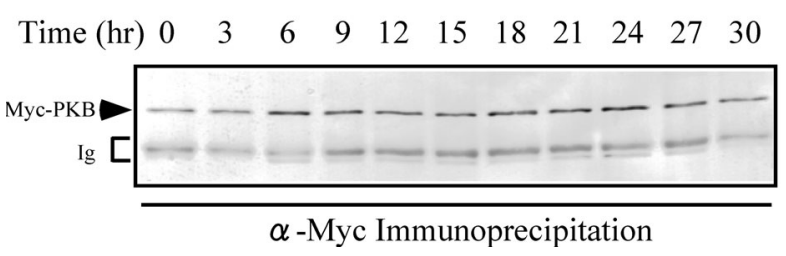

Fig. 4. Kinase activity of PKB during progesterone-induced oocyte maturation. (A) Changes in the kinase activity of Myc-WT PKB during progesterone-induced oocyte maturation. Oocytes were injected with $50 \mathrm{ng}$ of Myc-WT PKB mRNA and incubated overnight. At the indicated times after treatment with $10 \mu \mathrm{M}$ progesterone, 10 oocytes were extracted and immunoprecipitated with anti-Myc antibody. The PKB kinase activity associated with the immunoprecipitates was measured as described in Materials and Methods. As positive and negative controls, oocytes were injected with $50 \mathrm{ng}$ of Myc- $\Delta \mathrm{PH}$ TS PKB (TS) or Myc- $\triangle$ PH KTS PKB (KTS) mRNA, respectively, and the kinase activity was also measured after anti-Myc immunoprecipitation. Each value is the mean \pm SEM of values from three experiments. (B) Anti-Myc immunoblots of the immunoprecipitates used in (A) to confirm that the expression levels of Myc-WT PKB (Myc-PKB) in the oocytes were the same. Ig, immunoglobulins. 
protein, of which the molecular mass is comparable to that estimated from the cDNA sequence of Xenopus PKB (ca. 56 $\mathrm{kDa}$ ). The $60-\mathrm{kDa}$ protein was also detected in the anti-PKB immunoprecipitates from immature and mature oocyte extracts in both species (Fig. 2B). We therefore conclude that the $60-\mathrm{kDa}$ protein recognized by the antibody is an amphibian PKB.

Using the newly produced antibody, we examined the behavior of PKB during Rana oocyte maturation. PKB was detected throughout the process of oocyte maturation, with no remarkable changes in its protein contents (Fig. 2C).

\section{Expression of constitutively active PKB in oocytes}

To investigate the function of PKB for oocyte maturation, we investigated whether GVBD can be induced without progesterone stimulation by artificial expression of a constitutively active PKB in the oocytes. Two active phosphorylation sites of PKB, T309 and S474, were mutated to glutamic acid to construct a constitutively active PKB (TS PKB). In addition, $\mathrm{K} 180$ in the kinase domain was mutated to alanine to construct a kinase-deficient version (KTS PKB). mRNAs encoding these proteins were injected into Rana oocytes, but they failed to be expressed (data not shown). The reason for their failure to be expressed is not known. Then we constructed other mutants in which the pleckstrin homology $(\mathrm{PH})$ domain was removed from the full-length mutant PKBs ( $\triangle \mathrm{PH}$ TS $\mathrm{PKB}$ and $\triangle \mathrm{PH}$ KTS PKB). In contrast to the fulllength mutant PKBs, $\triangle \mathrm{PH}$ TS PKB and $\triangle \mathrm{PH} K \mathrm{KTS} P K B$ were successfully expressed in the oocytes when mRNAs were injected, and their protein concentration was higher than that of endogenous PKB (Fig. 3A). However, the overexpression of $\triangle \mathrm{PH}$ TS PKB, as well as that of $\triangle \mathrm{PH} K T S \mathrm{PKB}$, did not induce GVBD in the absence of progesterone.

Theoretically, $\triangle \mathrm{PH}$ TS $\mathrm{PKB}$ is constitutively active (Coffer et al., 1998); however, we suspected that it might be inactive in the oocytes, thereby failing to induce GVBD. To
A

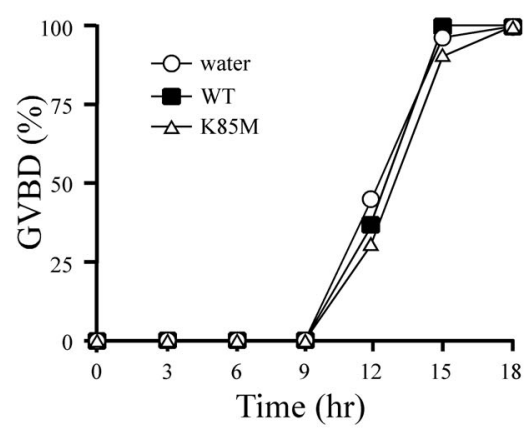

D

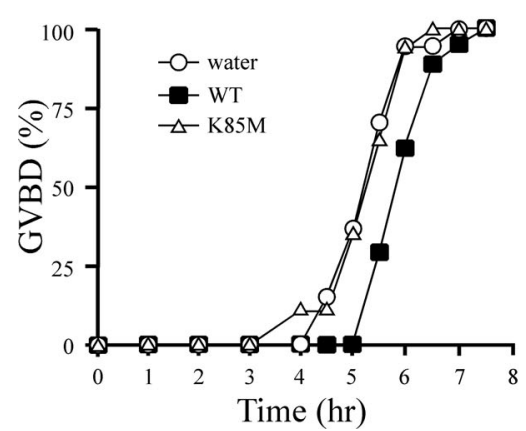

B

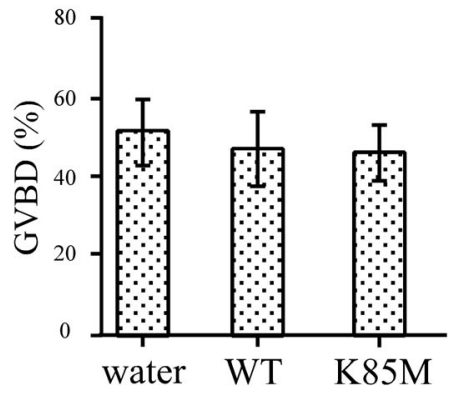

$\mathbf{E}$

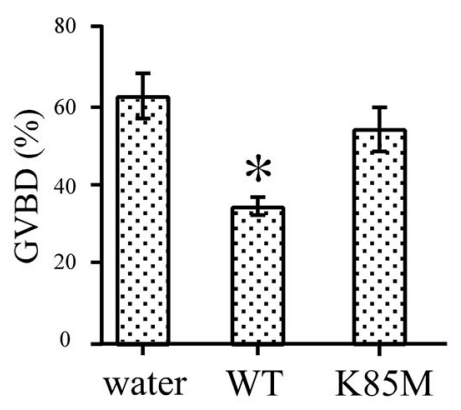

C

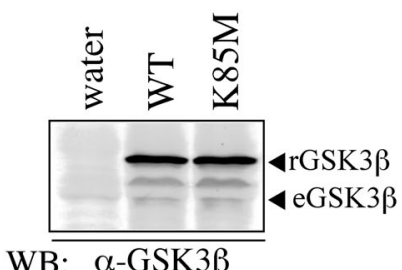

WB: $\alpha$-GSK3 $\beta$

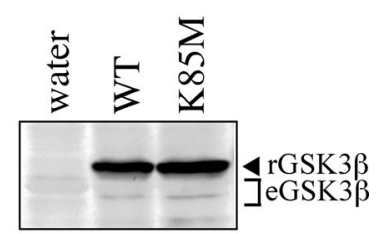

WB: $\alpha$-GSK3 $\beta$

Fig. 5. Effects of overexpression of GSK3 3 on oocyte maturation in Rana (A-C) and Xenopus (D-F). (A) Rana oocytes were injected with water or mRNA encoding wild-type (WT) or kinase-deficient (K85M) GSK3 3 . After overnight incubation, the injected oocytes were treated with $10 \mu \mathrm{M}$ progesterone and examined for the presence of GVBD at appropriate intervals. Representative data are shown. (B) Percentage of GVBD in oocytes overexpressing WT or K85M GSK3 $\beta$. The data were obtained when the control water-injected oocytes showed $50 \%$ GVBD, and are shown as the mean \pm SEM of values from four experiments. (C) Immunoblots of extracts from Rana oocytes injected with water or mRNAs encoding WT or K85M GSK3 $\beta$, indicating that the level of recombinant proteins (rGSK3 $\beta$ ) was higher than that of endogenous GSK3 $\beta$ (eGSK3 $\beta$ ). Extract equivalent to one oocyte was loaded to each lane and immunoblotted with anti-GSK3 $\beta$ antibody. (D) Xenopus oocytes were injected with water or mRNA encoding WT or K85M GSK3 $\beta$, incubated overnight, and stimulated with progesterone. The presence of GVBD was examined at appropriate intervals. Representative data are shown. (E) Percentage of GVBD in the GSK3 3 -overproducing Xenopus oocytes at the time when the control water-injected oocytes showed $\sim 50 \%$ GVBD. The values are the mean \pm SEM of values from three experiments. Overexpression of WT GSK3 $\beta$ delayed progesterone-induced Xenopus oocyte maturation (indicated by an asterisk, $P<0.05$, Student's $t$-test), but overexpression of K85M GSK3 $\beta$ had no effect. (F) Anti-GSK3 $\beta$ immunoblots of extracts from Xenopus oocytes injected with water or mRNA for WT or K85M GSK3 $\beta$. Extract equivalent to one oocyte was loaded to each lane. Note that recombinant GSK3 $\beta$ (rGSK3 $\beta$ ) is expressed at higher concentration than endogenous GSK3 $\beta$ (eGSK3 $\beta$ ). 
verify this possibility, we measured the kinase activity of $\triangle \mathrm{PH}$ TS PKB (Fig. 3B). The results clearly showed that $\triangle \mathrm{PH}$ TS PKB had activity over 172-fold higher than that of $\Delta \mathrm{PH}$ KTS PKB, indicating that the constitutively active $P K B$ is actually active in the oocytes but that its overexpression alone cannot induce oocyte maturation.

\section{PKB kinase activity during oocyte maturation}

Finally, we investigated whether endogenous PKB is activated during progesterone-induced Rana oocyte maturation. Because the anti-PKB antibody cannot precipitate PKB activity, we were unable to measure the kinase activity of endogenous PKB directly. Therefore, we injected oocytes with mRNA encoding a Myc-tagged version of wild-type PKB (Myc-WT PKB) and examined changes in its kinase activity during oocyte maturation after recovery of the Myc-tagged PKB by anti-Myc antibody. The kinase activity of Myc-WT PKB did not change significantly during the process of progesterone-induced oocyte maturation (Fig. 4A), in spite of its relatively constant protein level in each immunoprecipitate (Fig. 4B).

\section{Involvement of GSK3 $\beta$ in Rana oocyte maturation}

In Xenopus oocytes, it has been reported that GSK3 $\beta$ is inactivated during oocyte maturation, and that overexpression of GSK3 $\beta$ inhibits progesterone-induced oocyte maturation (Fisher et al., 1999; Sarkissian et al., 2004). Thus, we performed overexpression experiments to examine the involvement of GSK3 $\beta$ in Rana oocyte maturation. When Rana and Xenopus oocytes were injected with mRNA encoding a wild-type or a kinase-deficient version of GSK3 $\beta$, the oocytes produced the recombinant proteins, the amount of which was significantly greater than that of endogenous GSK3 $\beta$ (Fig. 5C, F). Consistent with the results previously reported (Fisher et al., 1999), the time course of oocyte maturation was retarded when wild-type GSK3 $\beta$, but not kinasedeficient GSK3 $\beta$, was injected into Xenopus oocytes (Fig. $5 \mathrm{D}, \mathrm{E})$, indicating that the recombinant wild-type GSK3 $\beta$ is functional in oocytes. However, the overexpression of wildtype GSK3 $\beta$, as well as kinase-deficient GSK3 $\beta$, did not inhibit Rana oocyte maturation (Fig. 5A, B).

\section{DISCUSSION}

In starfish, the PI3K/PKB-mediated signal transduction system has been reported to play a key role in oocyte maturation induced by 1-methyladenine, an $\mathrm{MIH}$ in starfish (Okumura et al., 2002). The involvement of PI3K in oocyte maturation in Rana dybowskii has also been suggested (Ju et al., 2002). In Xenopus, however, the requirement of PI3K in progesterone-induced oocyte maturation has been controversial, some studies indicating that activation of PI3K is required for the effects of progesterone and other studies indicating that it is not required (Muslin et al., 1993; LopezHernandez et al., 1999; Hehl et al., 2001). Concerning a downstream target of $\mathrm{PI} 3 \mathrm{~K}$, it has been reported that artificial introduction of PKB in Xenopus oocytes induced maturation without progesterone stimulation (Andersen et al., 1998), but it has also been suggested that PKB plays only an ancillary role during progesterone-induced oocyte maturation in Xenopus (Andersen et al., 2003). GSK3 $\beta$, a target of $\mathrm{PKB}$, is thought to be a negative regulator of Xenopus oocyte maturation, since oocyte maturation is delayed by overexpression of GSK3 $\beta$ (Fisher et al., 1999). These results suggest that the PI3K/PKB/GSK3 $\beta$-mediated signal transduction system has a role in induction of oocyte maturation in Xenopus, although it remains to be elucidated whether the system plays a key role in progesteroneinduced 'normal' oocyte maturation.

Although Xenopus is the most widely used experimental animal in amphibians, this species is atypical, at least in oocyte maturation; inactive MPF (pre-MPF) is present in Xenopus but not in any other amphibians, including Rana (Tanaka and Yamashita, 1995). Indeed, it has been demonstrated that the molecular mechanisms of the formation and activation of MPF in Rana are different from those in Xenopus (Yamashita et al., 2000; Yoshida et al., 2000b). In this study using Rana japonica, we investigated the involvement of PI3K, PKB, and GSK3 $\beta$ in oocyte maturation in response to progesterone, a natural hormone responsible for inducing oocyte maturation in amphibians. The following findings obtained in this study suggest the involvement of the PI3K pathway in the early signal transduction in Rana oocyte maturation: 1) The PI3K inhibitors wortmannin and LY294002 inhibited progesterone-stimulated GVBD, and 2) the inhibition was observed only when the drug was added in the early phase of oocyte maturation. However, results suggesting that PKB and GSK3 $\beta$ is not involved in induction of Rana oocyte maturation were obtained: 1) A constitutively active PKB $(\triangle \mathrm{PH}$ TS PKB) did not induce GVBD in the absence of progesterone, despite its high level of kinase activity in the oocytes, 2) the kinase activity of PKB measured by using Myc-tagged wild-type PKB (Myc-WT PKB) as an indicator did not change significantly during the process of progesterone-induced oocyte maturation, and 3) overexpression of GSK3 $\beta$ had no apparent effect on Rana oocyte maturation. Our findings strongly suggest that $\mathrm{PI} 3 \mathrm{~K}$ is essential for induction of Rana oocyte maturation, but that neither PKB nor GSK3 $\beta$ is a downstream mediator of the PI3K signaling pathway. What, then, is a downstream component of the PI3K pathway? It is generally known that a downstream mediator of $\mathrm{PI} 3 \mathrm{~K}$ is $\mathrm{PKB}$ and that $\mathrm{PKB}$, in turn, targets $p 70^{s 6 \mathrm{k}}$ as well as GSK3 $\beta$. The activity of $p 70^{\mathrm{s} 6 \mathrm{k}}$ increases during progesterone-induced maturation in Rana, and the suppression of its activity by rapamycin inhibits oocyte maturation (Bandyopadhyay et al., 1999). In addition, inhibition of PI3K leads to the suppression of $\mathrm{p} 70^{\mathrm{s} 6 \mathrm{k}}$ activity (Ju et al., 2002). These findings suggest that $p 70^{s 6 k}$ acts downstream of PI3K. Our findings exclude the possibility that $\mathrm{PKB}$ and $\mathrm{GSK} 3 \beta$ pass the signal from PI3K on to $\mathrm{p} 70^{\mathrm{s} 6 \mathrm{k}}$, but further studies are required to identify the mediators that function between PI3K and p70 ${ }^{\text {s6k }}$.

In Xenopus, inactivation of GSK3 $\beta$ induces oocyte maturation by stimulating the de-novo synthesis of Mos followed by the activation of MAPK and, conversely, overexpression of GSK3 $\beta$ delays the progress of oocyte maturation (Fisher et al., 1999, Sarkissian et al., 2004). In the present study, however, we have shown that overexpression of GSK3 $\beta$ has no effect on Rana oocyte maturation. This discrepancy may be attributable to a difference in the mechanisms of MPF activation between Rana and Xenopus oocyte maturation. Pre-MPF is present in Xenopus oocytes and is activated by MAPK, resulting in initiation of oocyte maturation (Yamashita, 
2000; Haccard and Jessus, 2006; Nagahama and Yamashita, 2008). In contrast to Xenopus, Rana oocytes do not have pre-MPF, and MAPK activation is not essential for the initiation of Rana oocyte maturation, although MAPK is activated in accordance with MPF activation during oocyte maturation (Tanaka and Yamashita, 1995; Yoshida et al., 2000a). Thus, it is highly likely that GSK3 $\beta$ functions to activate MAPK but not MPF; therefore, its activity is not required to initiate Rana oocyte maturation.

In summary, we have obtained results suggesting that PI3K, but not PKB or GSK3 $\beta$, is involved in induction of progesterone-induced oocyte maturation in Rana japonica. Further studies, including identification of molecules that link PI3K and Cyclin B translation, should enable us to understand the entire process of the signal transduction pathway from hormonal stimulation on the oocyte surface to the activation of MPF in the oocyte cytoplasm during progesteroneinduced oocyte maturation in Rana. In addition, comparative studies using Xenopus and Rana oocytes should provide a deeper insight into the generality and the specificity of the signal transduction system responsible for induction of progesterone-induced oocyte maturation in amphibians.

\section{ACKNOWLEDMENTS}

This work was supported in part by a grant-in-aid for scientific research (14340258) to M.Y., and by the 21st Century COE Program "Center of Excellence for Advanced Life Science on the Base of Bioscience and Nanotechnology", funded by the Ministry of Education, Science, Sports and Culture of Japan. We thank Prof. Yoshitaka Nagahama and Dr. Hiroko Kajiura-Kobayashi (NIBB, Okazaki, Japan) for sequencing the $\mathrm{N}$-terminal amino acids of $E$. coli-produced Xenopus PKB and for preparing the AKTide-2T peptide. All animal experiments in this study were approved by the Committee on Animal Experimentation, Hokkaido University.

\section{REFERENCES}

Alessi DR, Cohen P (1998) Mechanism of activation and function of protein kinase B. Curr Opin Genet Dev 8: 55-62

Andersen CB, Roth RA, Conti M (1998) Protein kinase B/Akt induces resumption of meiosis in Xenopus oocytes. J Biol Chem 273: 18705-18708

Andersen CB, Sakaue H, Nedachi T, Kovacina KS, Clayberger C, Conti M, Roth RA (2003) Protein kinase B/Akt is essential for the insulin- but not progesterone-stimulated resumption of meiosis in Xenopus oocytes. Biochem J 369: 227-238

Bandyopadhyay A, Bandyopadhyay J, Chung J, Choi HS, Kwon HB (1999) Inhibition of S6 kinase by rapamycin blocks maturation of Rana dybowskii oocytes. Gen Comp Endocrinol 113: 230239

Bagowski CP, Myers JW, Ferrell JE (2001) The classical progesterone receptor associates with p42 MAPK and is involved in phosphatidylinositol 3-kinase signaling in Xenopus oocytes. J Biol Chem 276: 37708-37714

Carnero A, Lacal JC (1998) Wortmannin, an inhibitor of phosphatidylinositol 3-kinase, induces oocyte maturation through a MPFMAPK-dependent pathway. FEBS Lett 422: 155-159

Coffer PJ, Jin J, Woodgett JR (1998) Protein kinase B (c-Akt): a multifunctional mediator of phosphatidylinositol 3-kinase activation. Biochem J 335: 1-13

Cross DAE, Alessi DR, Cohen P, Andjelkovich M, Hemmings BA (1995) Inhibition of glycogen synthase kinase-3 by insulin mediated by protein kinase B. Nature 378: 785-789

Cyert MS, Kirschner MW (1988) Regulation of MPF activity in vitro. Cell 53: 185-195
Ferrell JE Jr (1999) Xenopus oocyte maturation: new lessons from a good egg. BioEssays 21: 833-842

Fisher DL, Morin N, Dorée M (1999) A novel role for glycogen synthase kinase-3 in Xenopus development: maintenance of oocyte cell cycle arrest by a $\beta$-catenin-independent mechanism. Development 126: 567-576

Haccard O, Jessus C (2006) Oocyte maturation, Mos and cyclinsa matter of synthesis: two functionally redundant ways to induce meiotic maturation. Cell Cycle 5: 1152-1159

Hehl S, Stoyanov B, Oehrl W, Schonherr R, Wetzker R, Heinemann $\mathrm{SH}$ (2001) Phosphoinositide 3-kinase- $\gamma$ induces Xenopus oocyte maturation via lipid kinase activity. Biochem J 360: 691698

Hirai T, Yamashita M, Yoshikuni M, Lou YH, Nagahama Y (1992) Cyclin B in fish oocytes: its cDNA and amino acid sequences, appearance during maturation, and induction of p34 ${ }^{\text {cdc2 }}$ activation. Mol Reprod Dev 33: 131-140

Ihara J, Yoshida N, Tanaka T, Mita K, Yamashita M (1998) Either cyclin B1 or B2 is necessary and sufficient for inducing germinal vesicle breakdown during frog (Rana japonica) oocyte maturation. Mol Reprod Dev 50: 499-509

Ju JW, Bandyopadhyay A, Im WB, Chung J, Kwon HB, Choi HS (2002) Involvement of phosphatidylinositol 3 kinase in the progesterone-induced oocyte maturation in Rana dybowskii. Gen Comp Endocrinol 126: 213-220

Kotani T, Yamashita M (2002) Discrimination of the roles of MPF and MAP kinase in morphological changes that occur during oocyte maturation. Dev Biol 252: 271-286

Kotani T, Yoshida N, Mita K, Yamashita M (2001) Requirement of cyclin B2, but not cyclin B1, for bipolar spindle formation in frog (Rana japonica) oocytes. Mol Reprod Dev 59: 199-208

Lopez-Hernandez E, Santos E (1999) Oncogenic Ras-induced germinal vesicle breakdown is independent of phosphatidylinositol 3-kinase in Xenopus oocytes. FEBS Lett 451: 284-288

Masui $Y$ (1985) Meiotic arrest in animal oocytes. Biol Fertil 1: 189219

Muslin AJ, Klippel A, Williams LT (1993) Phosphatidylinositol 3kinase activity is important for progesterone-induced Xenopus oocyte maturation. Mol Cell Biol 13: 6661-6666

Nakahata S, Kotani T, Mita K, Kawasaki T, Katsu Y, Nagahama Y, Yamashita M (2003) Involvement of Xenopus Pumilio in the translational regulation that is specific to cyclin B1 mRNA during oocyte maturation. Mech Dev 120: 865-880

Nagahama Y, Yamashita M (2008) Regulation of oocyte maturation in fish. Dev Growth Differ 50: S195-S219

Nagahama Y, Yoshikuni M, Yamashita M, Tokumoto T, Katsu Y (1995) Regulation of oocyte growth and maturation in fish. Curr Top Dev Biol 30: 103-145

Nebreda AR, Ferby I (2000) Regulation of the meiotic cell cycle in oocytes. Curr Opin Cell Biol 12: 666-675

Obata T, Yaffe MB, Leparc GG, Piro ET, Maegawa H, Kasiwagi A, Kikkawa R, Cantley LC (2000) Peptide and protein library screening defines optimal substrate motifs for AKT/PKB. J Biol Chem 275: 36108-36115

Okumura E, Fukuhara T, Yoshida H, Hanada S, Kozutsumi R, Mori M, Tachibana K, Kishimoto T (2002) Akt inhibits Myt1 in the signalling pathway that leads to meiotic G2/M-phase transition. Nat Cell Biol 4: 111-116

Palmer A, Nebreda AR (2000) The activation of MAP kinase and p34cdc2/cyclin B during the meiotic maturation of Xenopus oocytes. Prog Cell Cycle Res 4: 131-143

Sakamoto I, Takahara K, Yamashita M, Iwao Y (1998) Changes in cyclin B during oocyte maturation and early embryonic cell cycle in the newt, Cynops pyrrhogaster: requirement of germinal vesicle for MPF activation. Dev Biol 195: 60-69

Sarkissian M, Mendez R, Richter JD (2004) Progesterone and insulin stimulation of CPEB-dependent polyadenylation is regu- 
lated by Aurora A and glycogen synthase kinase-3. Genes Dev 18: $48-61$

Shtivelman E, Sussman J, Stokoe D (2002) A role for PI 3-kinase and PKB activity in the G2/M phase of the cell cycle. Curr Biol 12: 919-924

Tanaka T, Yamashita M (1995) Pre-MPF is absent in immature oocytes of fishes and amphibians except Xenopus. Dev Growth Differ 37: 387-393

Vanhaesebroeck B, Alessi DR (2000) The PI3K-PDK1 connection: more than just a road to PKB. Biochem J 346: 561-576

Yamashita M (1998) Molecular mechanisms of meiotic maturation and arrest in fish and amphibian oocytes. Semin Cell Dev Biol 9: $569-579$

Yamashita M (2000) Toward modeling of a general mechanism of MPF formation during oocyte maturation in vertebrates. Zool Sci 17: 841-851
Yamashita M, Yoshikuni M, Hirai T, Fukada S, Nagahama Y (1991) A monoclonal antibody against the PSTAIR sequence of p34 ${ }^{\text {cdc2 }}$, catalytic subunit of maturation-promoting factor and key regulator of the cell cycle. Dev Growth Differ 33: 617-624

Yamashita M, Mita K, Yoshida N, Kondo T (2000) Molecular mechanisms of the initiation of oocyte maturation: General and species specific aspects. Prog Cell Cycle Res 4: 115-129

Yoshida N, Mita K, Yamashita M (2000a) The function of the Mos/ MAPK pathway during oocyte maturation in the Japanese brown frog Rana japonica. Mol Reprod Dev 57: 88-98

Yoshida N, Mita K, Yamashita M (2000b) Comparative study of the molecular mechanisms of oocyte maturation in amphibians. Comp Biochem Physiol B 126: 189-197

(Received April 14, 2008 / Accepted May 6, 2008) 